\title{
Wavefront analysis of a white-light supercontinuum
}

\author{
Emma Kueny, ${ }^{1,2,{ }^{*}}$ Joachim Meier, ${ }^{1,2}$ Xavier LevecQ, ${ }^{3}$ \\ NADEZdA VARKENTINA, ${ }^{3}$ FRANZ X. KÄRTNER, ${ }^{1,2,4}$ AND \\ AnNe-LAURE CALENDRON ${ }^{1,4}$ \\ ${ }^{1}$ Center for Free-Electron Laser Science (CFEL), Deutsches Elektronen Synchrotron (DESY), Notkestraße \\ 85, 22607 Hamburg, Germany \\ ${ }^{2}$ Department of Physics, Universität Hamburg, Luruper Chaussee 149, 22761 Hamburg, Germany \\ ${ }^{3}$ Imagine Optic, 18 Rue Charles de Gaulle, 91400 Orsay, France \\ ${ }^{4}$ The Hamburg Centre for Ultrafast Imaging, Universität Hamburg, Luruper Chaussee 149, \\ 22761 Hamburg, Germany \\ *emma.kueny@cfel.de
}

\begin{abstract}
The wavefront quality of white-light supercontinuum is crucial for applications like waveform synthesis or imaging. It has been here generated by $1030 \mathrm{~nm}$ centered sub-picosecond pulses in YAG and characterized with a Shack-Hartmann wavefront sensor across different parts of the spectrum. It shows a good wavefront quality of $\lambda / 11$ and little dependence on the wavelength of the supercontinuum. The wavefront deformations are transferred from the driver laser wavefront to the supercontinuum independently of the wavelength.
\end{abstract}

(C) 2018 Optical Society of America under the terms of the OSA Open Access Publishing Agreement

\section{Introduction}

White-light supercontinuum generation is the wide spectral broadening towards shorter and longer wavelengths of a laser beam propagating through a material, due to several nonlinear effects occurring at high intensity. In the case of ultrashort pulses, the initial phenomenon involved is self-phase modulation, in which the laser pulse modulates its own phase due to the Kerr-nonlinearity of the material [1], leading to self-steepening and self-focusing. Among other important mechanisms is plasma generation, leading also to defocusing $[2,3]$. The equilibrium between self-focusing and plasma defocusing generates a single or multiple filaments.

Supercontinua were first reported in $1970[4,5]$ and have become of widespread use in ultrafast laser systems, enabling developments in ultrafast laser spectroscopy [6], imaging [7], and generation of single to few cycles laser sources. In the latter, the white-light supercontinuum is used for example to seed optical parametric amplifiers $[8,9]$. Particularly in this case, a good spatial beam quality is crucial: the beam quality of the final waveform depends on that of the white-light and on its amplification. To the best of our knowledge, there has been only one study of the wavefront of white-light supercontinua generated in fibers [10] and no systematic experimental characterization of the wavefront of white-light generated in bulk, due to the difficulty of measuring broadband wavefronts.

Two common wavefront measurement methods are Shack-Hartmann sensors and lateral shearing interferometers. The principles behind the reconstruction are the same [11] and each method involves a trade-off between the dynamic range, the resolution, the bandwidth and the practicality of use. A comparison of the performances of Shack-Hartmann and achromatic three-wave and quadri-wave lateral shearing interferometers can be found in [12]. As the latter depends on the use of a grating, its bandwidth is restricted to one octave due to the overlap between adjacent diffraction orders, and the dynamic range of the measurement is reduced away from the frequency for which the interferometer is optimized. By contrast, the bandwidth of Shack-Hartmann wavefront sensors is mainly limited by the chromatic aberrations of the lenses [13]. For this experiment, we took advantage of the disponibility of a broadband 
Shack-Hartmann sensor with achromatic lenses, calibrated over the full spectral range of its silicon CCD camera ( $400 \mathrm{~nm}$ to $1100 \mathrm{~nm}$ ).

In this paper, we report on a systematic experimental study of the blue wing of a white-light supercontinuum generated by $1030 \mathrm{~nm}$, sub-picosecond laser pulses in YAG. We measured with a broadband Shack-Hartmann wavefront sensor the wavefront of the white-light and studied its spectral dependency. Our analysis includes the artifacts which are introduced during the acquisition process, and investigates the relationship between the wavefronts of white-light supercontinua and driver laser beams.

\section{Experimental setup and analysis}

We used a $1030 \mathrm{~nm}$ diode-pumped solid-state $\mathrm{Yb}: \mathrm{KYW}$ source comprising oscillator, stretcher, regenerative amplifier and compressor and providing $650 \mathrm{fs}$ long, $5.5 \mathrm{~mJ}$ pulses at $1 \mathrm{kHz}$. The energy fluctuations are under $1 \%$ pulse-to-pulse, as well as measured over long term [14]. At the input of the white-light setup, the beam is collimated to $1 \times 1.7 \mathrm{~mm}^{2}$ [15]. The white-light was generated by focusing a fraction of the energy (a few $\mu \mathrm{J}$ ) into a $10 \mathrm{~mm}$ long YAG rod, with the same experimental parameters as described in [9]: the focusing lens has $100 \mathrm{~mm}$ focal length, and an iris was used to fine tune the input beam diameter to reach a stable white-light continuum.

(a)

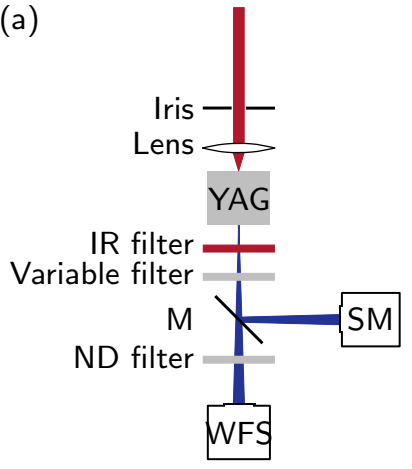

(b)

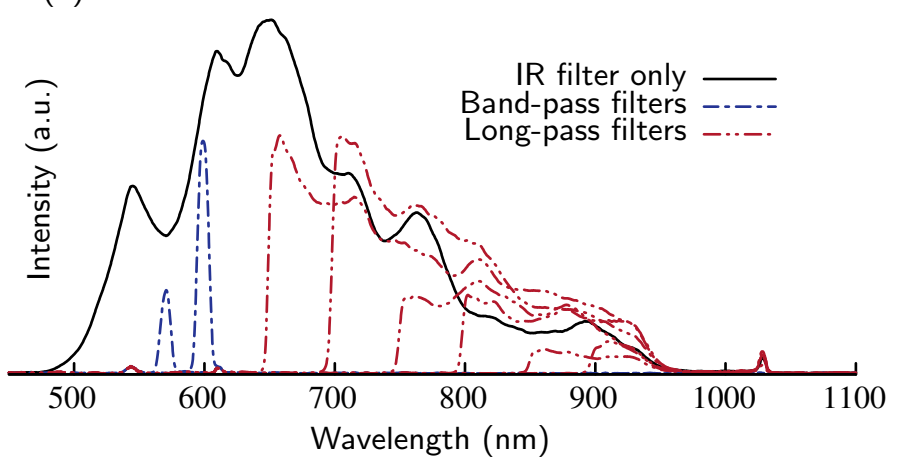

Fig. 1. (a) Setup of the experiment. Red: $1030 \mathrm{~nm}$ laser driver; blue: white-light. M: flip mirror; SM: spectrometer; WFS: wavefront sensor. (b) Average spectrum recorded for different variable filters.

As shown in Fig. 1(a), a $950 \mathrm{~nm}$ short-pass filter (IR filter) was used in all measurements to filter out the infrared beam and transmit only the white-light. Its transmittance was measured to be $0.07 \%$ at $1030 \mathrm{~nm}$. A variable second filter allowed to select a part of the spectrum to be analyzed with the wavefront sensor. Two $20 \mathrm{~nm}$ wide band-pass filters centered at $570 \mathrm{~nm}$ and $600 \mathrm{~nm}$ allowed to study narrow spectral regions of the white-light while $650 \mathrm{~nm}$ to $900 \mathrm{~nm}$ long-pass filters permitted to progressively reduce the bandwidth. In addition, different combinations of neutral density filters were added to avoid saturation of the wavefront sensor. For each filter configuration, the spectrum of the transmitted spectral band (Fig. 1(b)) and single-shot wavefronts were recorded. The used fiber-based spectrometer is an OceanOptics HR 4000 covering $200 \mathrm{~nm}$ to $1100 \mathrm{~nm}$, with a resolution of less than $1 \mathrm{~nm}$ full-width half-maximum; the light was directly coupled to the spectrometer. We used the HASO4 Broadband Shack-Hartmann sensor from Imagine Optic, allowing measurements over a broad spectral range from $400 \mathrm{~nm}$ to $1100 \mathrm{~nm}$ with an array size of $68 \times 50$. The spatial resolution of the Shack-Hartmann sensor is $105 \mu \mathrm{m}$. A small part of the $1030 \mathrm{~nm}$ beam is visible in the spectrum (Fig. 1(b)) and also reaches the wavefront sensor, but its intensity relative to the white-light is low enough to not influence the calculation of the wavefront. The wavefront sensor was located $\sim 11 \mathrm{~cm}$ after the crystal with the neutral 
density filters mounted on its input opening. The colored filters were placed $1 \mathrm{~cm}$ to $2 \mathrm{~cm}$ after the crystal.

The analysis of the recorded wavefronts was performed with Imagine Optics' WaveView software. The horizontal and vertical tilt and defocus components of each wavefront were automatically subtracted from the zonal reconstitution in order to observe only the wavefront aberrations. In addition, the wavefronts were decomposed on a basis of 210 Zernike polynomials [16], fitted on a circular pupil of identical radius, to allow a quantitative comparison of specific aberrations. The absorption of all used filters have been separately measured in the relevant spectral range with a PerkinElmer spectrometer. Their induced aberrations have also been separately characterized by interferometry with a $632.8 \mathrm{~nm}$ continuous-wave laser. In order to compare the wavefront of the white-light with the wavefront of the driving laser, this latter has been recorded with the wavefront sensor placed between the lens and the crystal and numerically propagated up to the interaction point.

\section{Zonal wavefronts}

Figure 2 presents one single-shot zonal wavefront for each configuration. The subfigures 2(a) and 2(b) correspond to $20 \mathrm{~nm}$-large bandwidths centered at $570 \mathrm{~nm}$ and $600 \mathrm{~nm}$, while the subfigures (c) to (h) show an increasingly large bandwidth up to $950 \mathrm{~nm}$, and the subfigure (i) is the full visible wing of the white-light. The wavefront aberrations were stable from shot to shot, as shown by the standard deviation of the Zernike coefficients in Fig. 3. The difference in the size of the wavefronts comes from the neutral density filters, as the wavefront sensor only reconstructs the wavefront where the beam had sufficient intensity.
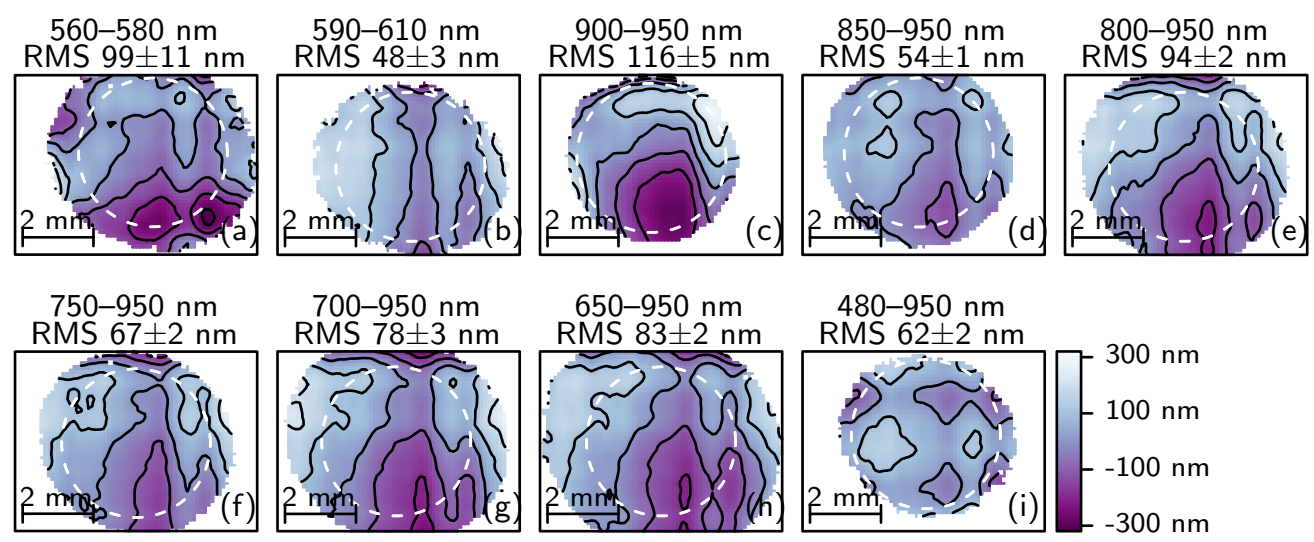

Fig. 2. Single-shot wavefronts of each bandwidth separately studied. The piston, tilt and defocus components of the wavefront are subtracted, leaving the wavefront aberrations centered around zero. Positive values correspond to a phase lag. The dashed circles indicate the part of the beam used in Section 4 for the decomposition into Zernike polynomials. The contours are separated by $100 \mathrm{~nm}$.

The shape is composed of a dip at the bottom and a crest on the top and the sides, which is observable between $900 \mathrm{~nm}$ and $950 \mathrm{~nm}$ on Fig. 2(c). It remains visible as the bandwidth increases and more structures are appearing in Figs. 2(d)-2(i), which can be described as vertical and horizontal lines in the wavefront. The dip is also present on the other side of the spectrum, at $570 \mathrm{~nm}$ (a). In contrast, the full wavefront (i), which is an intensity-weighted average of each spectral component due to the averaging of the local wavefront slope introduced by Shack-Hartmann wavefront sensors [11], does not show this bottom dip. This is also the case of the wavefront measured at $600 \mathrm{~nm}$, close to the intensity maximum (see Fig. 1). The fact 
that the wavefront measured between $650 \mathrm{~nm}$ and $950 \mathrm{~nm}$ (Fig. 2(h)) presents a shape similar to both wavefronts measured around $600 \mathrm{~nm}$ (Fig. 2(b)) and between $900 \mathrm{~nm}$ and $950 \mathrm{~nm}$ (Fig. 2(c)) seems to show that the wavefront is smoothly evolving with the wavelength. This is not surprising, as it has been shown that the white-light retains a stable phase relationship with its driver across the whole spectrum [17].

The quality of each wavefront can be defined by the root mean square (RMS) deviation from a flat surface. This quantity, averaged over 50 successive wavefront measurements, is indicated for each configuration on Fig. 2. The RMS is spanning from (48 \pm 2$) \mathrm{nm}$ to $(116 \pm 3) \mathrm{nm}$ without specific trend and seems unrelated to the neutral density filters that were used and could have influenced the wavefront measurements. Considering a central wavelength for the full beam of $\lambda=700 \mathrm{~nm}$, the complete wavefront has an RMS distortion of $\lambda / 11$, which is a good quality according to the Maréchal criterion (the Maréchal criterion states that a beam is considered diffraction limited when its wavefront deformation is under $\lambda / 14$ [18]).

\section{Specific aberrations}

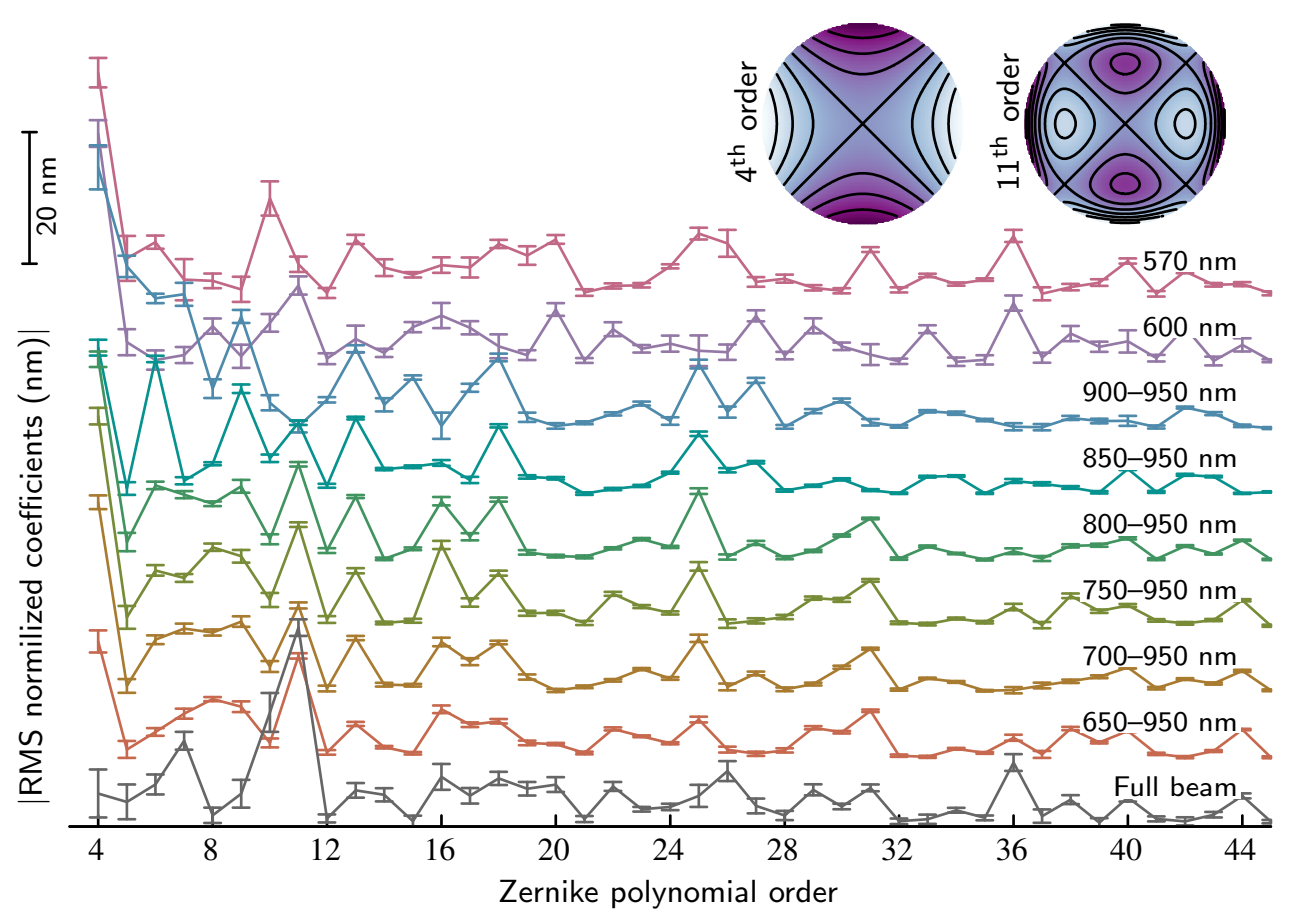

Fig. 3. Absolute value of the $4^{\text {th }}$ to $45^{\text {th }}$ Zernike coefficients with RMS normalization, in the order given by WaveView. The coefficients are averaged over 30 shots; the error bars represent the standard deviation. Inserts: representation of the $4^{\text {th }}$ and $11^{\text {th }}$ Zernike polynomials.

In order to be quantified, the wavefront error of the white-light can be decomposed into specific aberrations. Zernike polynomials $[16,19]$ are widely used to represent wavefront aberrations of round beams. Figure 3 presents the absolute value of the coefficients of the first 45 Zernike polynomials averaged over 30 successive shots for each configuration. The polynomials 1 to 3 which are not shown represent the tilts and defocus of the beam. The polynomials are normalized so that each coefficient represents the contribution to the RMS deformation due to the associated aberration. The total RMS of each wavefront is then the sum of the coefficients of all polynomials. 
All coefficients have been calculated by fitting the wavefront on the largest common pupil radius $(2.1 \mathrm{~mm})$ in order to be comparable (see dashed circles on Fig. 2).

Since the measured wavefronts have a poor central symmetry, the decomposition in Zernike polynomials is expected to involve many high-order polynomials with small coefficients. Indeed, most polynomials are contributing less than $10 \mathrm{~nm}$ to the wavefront. Some polynomials have a higher coefficient for several filter configurations, confirming the shape similarities between the wavefronts.

In every case except the full beam, the main aberration is vertical astigmatism (the $4^{\text {th }}$ polynomial, of equation $\sqrt{6} r^{2} \cos 2 \theta$ in polar coordinates), ranging from $(17.6 \pm 1.7) \mathrm{nm}$ to $(39.6 \pm 3.3) \mathrm{nm}$ RMS. This is visible also by eye when comparing the wavefronts in Fig. 2 to the insert in Fig. 3. However, in the case of the full beam, the highest aberration is the vertical secondary astigmatism $\left(11^{\text {th }}\right.$ polynomial, $\left.\sqrt{10}\left(4 r^{2}-3\right) r^{2} \cos 2 \theta\right)$ with $29.7 \mathrm{~nm}$ RMS. This polynomial is similar to the $4^{\text {th }}$ except that it presents additional features on the outer part of the circle. These features, particularly the top left dip visible on Fig. 2(i), are visually similar to the ones on the measurement at $570 \mathrm{~nm}$ (Fig. 2(a)) while they don't show in the other wavefronts. This suggest they appear in the low wavelengths. The small coefficient of the vertical secondary astigmatism in the decomposition of the $570 \mathrm{~nm}$ wavefront can be explained by the chosen fitting surface (dashed line in Fig. 2): the whole wavefront of the full beam was encompassed by the pupil while the outer part of the other wavefronts was unused for the Zernike decomposition, leading to a higher coefficient of the $4^{\text {th }}$ polynomial. All other coefficients are globally decreasing with the order and have individually little influence on the shape of the wavefront.

\section{Divergence}

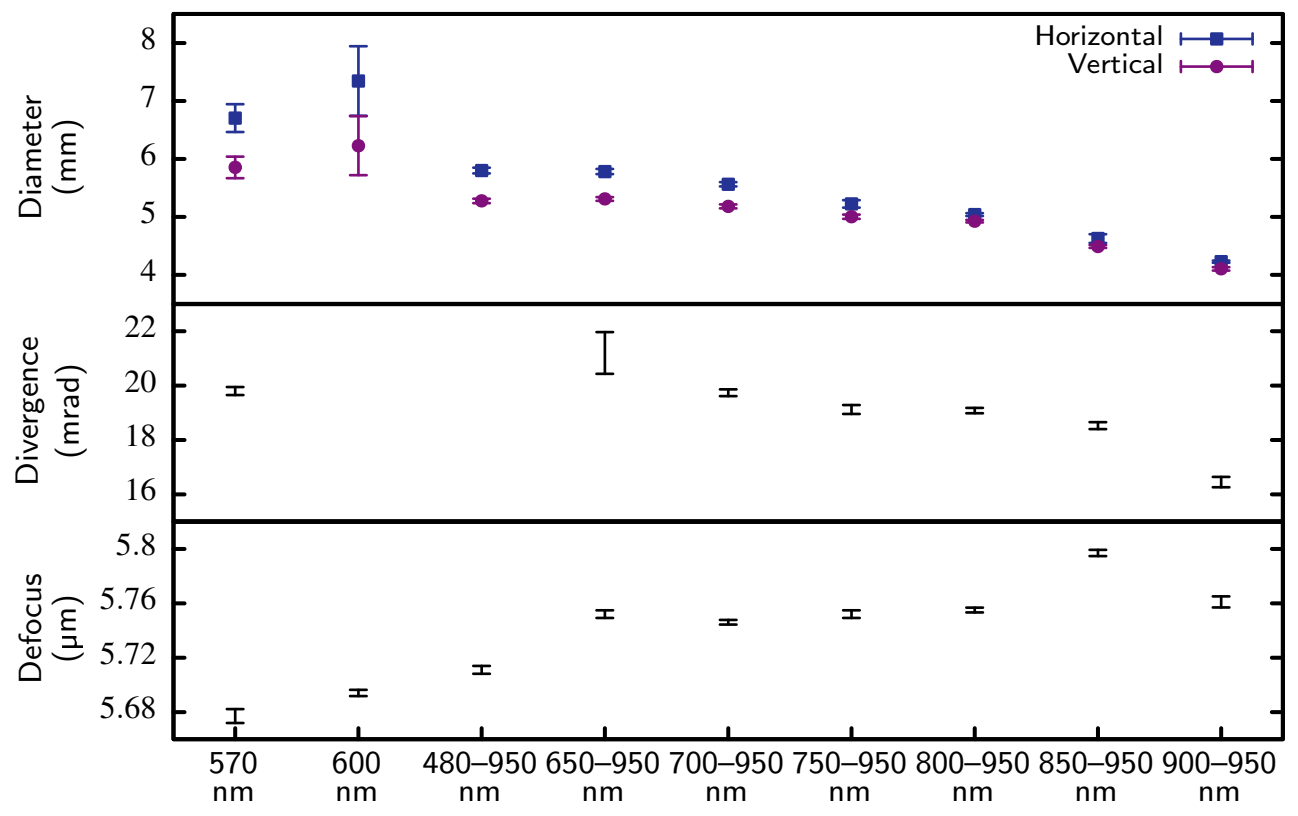

Fig. 4. $1 / e^{2}$ diameters and divergence of the white-light and coefficient of the Zernike polynomial corresponding to the curvature of its wavefront, in all measured transmitted bandwidth.

In addition to the wavefront, we recorded the intensity profile of the incident beam on the wavefront sensor. Figure 4 shows the $1 / e^{2}$ average beam diameter for each filter configuration. 
This diameter was calculated from the truncated intensity profiles recorded by the wavefront sensor, corrected by the maximal absorption of the neutral density filters at the corresponding wavelengths. One can see that the beam diameter is decreasing when the bandwidth is reduced from $480-950 \mathrm{~nm}$ to $900-950 \mathrm{~nm}$, indicating that the low wavelengths have a larger beam size. Notably, the components at 560-580 $\mathrm{nm}$ and 590-610 $\mathrm{nm}$ have a bigger diameter than the complete beam. This is explained by the fact that the full beam and the measurements from 650-950 nm to 750-950 nm were taken with a neutral density filter with an absorbance above 2 under $619 \mathrm{~nm}$ and between 1.2 and 1.4 above $716 \mathrm{~nm}$, thus cutting out the lower wavelengths.

Similarly, the divergence of the beam, which is calculated from the wavefront by WaveView, decreases as the wavelength increases, as reported in previous works [20,21]. However, the coefficient of the Zernike polynomial corresponding to the wavefront curvature $\left(\sqrt{2}\left(2 r^{2}-1\right)\right)$ stays almost constant in all configurations, indicating that the white-light is emitted from the same origin point over the whole spectrum, which has been previously reported to be at the beginning of the filament in the crystal [20,22].

\section{Origins of the deformations}

\subsection{Influence of the filters}

All acquisition configurations consisted of the white-light being transmitted through the $950 \mathrm{~nm}$ short-pass filter, another long-pass or band-pass filter, as well as up to two neutral density filters, each of which are adding some deformation to the wavefront of the white-light. Thus one possible origin of the measured wavefronts aberrations is the quality of the filters. To decouple the deformation coming from the filters from the wavefront deformation present in the white-light since its generation, the quality of some of the filters have been separately measured in transmission in a Michelson interferometer with a $632.8 \mathrm{~nm}$ helium-neon laser. The measured $950 \mathrm{~nm}$ short-pass filter as well as the 0.2 and 0.6 neutral density filters were the ones used during the study of the white-light, while the $500 \mathrm{~nm}$ and $550 \mathrm{~nm}$ long-pass filters were coming from the same lot as the previously used long-pass filters and can be assumed to have a similar quality than the ones used as variable filter in the experiment.

As can be seen in Fig. 5, the peak-to-valley wavefront of the measured filters is less than $122 \mathrm{~nm}$, and the highest measured wavefront RMS is $11.6 \mathrm{~nm}$, which is lower than the white-light wavefronts RMS. The reference wavefront when no filter was placed in the interferometer has an RMS of $5.33 \mathrm{~nm}$ and a peak-to-valley of $45 \mathrm{~nm}$, which means that a significant part of the wavefront distortions of the filters is measurement noise. Except in the case of the $550 \mathrm{~nm}$ long-pass filter, none of the measured wavefront has a shape which differs significantly from the reference. One can conclude that the filters were not responsible for the shape of the wavefront of the white-light and that they contributed little to the total wavefront RMS.

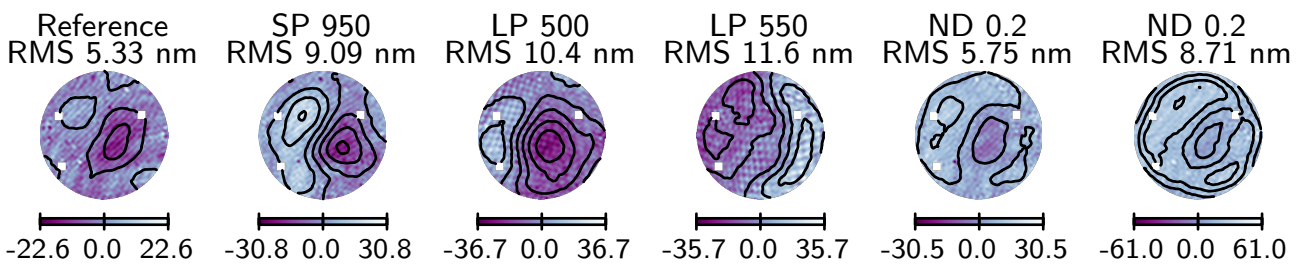

Fig. 5. Wavefront retrieved by interferometry of short-pass (SP), long-pass (LP) and neutral density (ND) filters as well as a reference wavefront with no filter. Some spots were coming from damages on the camera filters and have been occulted on all pictures before calculation of the RMS. The color scale is in nanometers. The contours are separated by $5 \mathrm{~nm}$. 


\subsection{Influence of the wavefront of the driving laser}

Another possible factor influencing the wavefront of the white-light is the wavefront of the infrared driver, due to their stable phase relationship [1,17]. The wavefront of the driving laser was measured with the wavefront sensor located between the focusing lens and the YAG crystal (see Fig. 1(a)), then numerically propagated $66 \mathrm{~mm}$ up to the focal point [23]. The resulting wavefront at the position of the crystal is shown in Fig. 6(d).
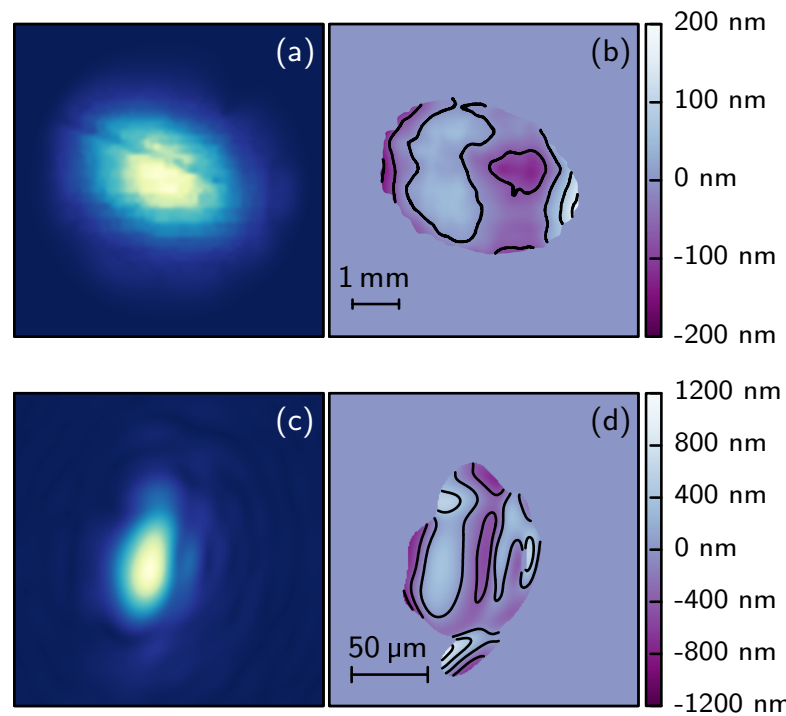

$1200 \mathrm{~nm}$
$800 \mathrm{~nm}$
$400 \mathrm{~nm}$
$0 \mathrm{~nm}$
$-400 \mathrm{~nm}$
$-800 \mathrm{~nm}$

Fig. 6. (a) Measured intensity profile and (b) wavefront of the infrared driver $66 \mathrm{~mm}$ before the crystal. (c) Intensity profile and (d) wavefront of the numerically propagated beam at the input surface of the crystal. Tilt and defocus components are subtracted from the wavefronts. The contours are separated by $75 \mathrm{~nm}$ (b) and $400 \mathrm{~nm}(\mathrm{~d})$.

The $1030 \mathrm{~nm}$ driver has significant (close to $2 \lambda$ peak-to-valley) aberrations in the focus, where it generates the white-light. The wavefront RMS is $296 \mathrm{~nm}$. Its shape presents similarities with the patterns in the white-light wavefront: positive values on the sides with a dip in the middle. The difference between the peak and the dip in the central part of the beam is on the order of $600 \mathrm{~nm}$, which corresponds to the peak-to-valley wavefront deformation of the white-light. This suggests that the wavefront of the infrared driver would be responsible for the wavefront distortions of the white-light.

\section{Conclusion}

The wavefront of white-light continuum, generated in bulk YAG, is measured here in the visible wing for the first time. In order to understand the dependency of the wavefront quality over the spectral extend of the white-light, we used band-pass and long pass-filters to reduce the spectral content of the white-light. The white-light wavefront exhibits over the full visible wing a good quality $\left(\lambda / 11\right.$ RMS) and its aberrations are mainly described by $0^{\circ}$ astigmatism. The deformation pattern and the wavefront quality remain about constant in all spectral regions. The most probable origin of the measured wavefront deformations is the wavefront of the $1030 \mathrm{~nm}$ laser driving the generation, measured experimentally before the focus and propagated numerically to the focus. The wavefront sensor was able to measure the increase of the white-light divergence with the frequency. This work demonstrates the importance of a good beam quality of the driver laser, in order to generate a white-light supercontinuum with high beam quality. We have also shown 
that broadband Shack-Hartmann wavefront sensors are a suitable tool for the characterization of supercontinua, and can presumably be used to optimize the generation process.

\section{Funding}

Helmholtz Association

\section{Acknowledgments}

Anne-Laure Calendron acknowledges a post-doc grant from the Helmholtz association.

\section{References}

1. R. R. Alfano, The supercontinuum laser source: the ultimate white light (Springer, 2016), 3rd ed.

2. N. Bloembergen, "The influence of electron plasma formation on superbroadening in light filaments," Opt. Commun. 8, 285-288 (1973).

3. A. M. Zheltikov, "Let there be white light: supercontinuum generation by ultrashort laser pulses," Physics-Uspekhi 49, 605-628 (2006).

4. R. R. Alfano and S. L. Shapiro, "Emission in the region 4000 to 7000 Å via four-photon coupling in glass," Phys. Rev. Lett. 24, 584-587 (1970).

5. R. R. Alfano and S. L. Shapiro, "Observation of self-phase modulation and small-scale filaments in crystals and glasses," Phys. Rev. Lett. 24, 592-594 (1970).

6. R. L. Fork, W. J. Tomlinson, C. V. Shank, C. Hirlimann, and R. Yen, "Femtosecond white-light continuum pulses," Opt. Lett. 8, 1-3 (1983).

7. D. Wildanger, E. Rittweger, L. Kastrup, and S. W. Hell, "STED microscopy with a supercontinuum laser source," Opt. Express 16, 9614-9621 (2008).

8. C. Manzoni, O. D. Mücke, G. Cirmi, S. Fang, J. Moses, S.-W. Huang, K.-H. Hong, G. Cerullo, and F. X. Kärtner, "Coherent pulse synthesis: towards sub-cycle optical waveforms," Laser \& Photonics Rev. 9, 129-171 (2015).

9. H. Çankaya, A.-L. Calendron, C. Zhou, S.-H. Chia, O. D. Mücke, G. Cirmi, and F. X. Kärtner, "40- $\mu$ J passively CEPstable seed source for ytterbium-based high-energy optical waveform synthesizers,” Opt. Express 24, 25169-25180 (2016).

10. D. Li, C. Zhao, W. Wu, and S. Du, "Measurement of the wavefront distribution characteristics of a 400-1700nm supercontinuum light source," in Proc. SPIE 10457, (2017), 104571W.

11. J. Primot, "Theoretical description of Shack-Hartmann wave-front sensor," Opt. Commun. 222, 81-92 (2003).

12. J.-C. Chanteloup, "Multiple-wave lateral shearing interferometry for wave-front sensing," Appl. Opt. 44, 1559-1571 (2005).

13. B. C. Platt and R. Shack, "History and principles of Shack-Hartmann wavefront sensing," J. Refract. Surg. 17, S573-S577 (2001).

14. A.-L. Calendron, H. Çankaya, and F. X. Kärtner, "High-energy kHz Yb:KYW dual-crystal regenerative amplifier," Opt. Express 22, 24752-24762 (2014).

15. A.-L. Calendron, H. Çankaya, G. Cirmi, and F. X. Kärtner, "White-light generation with sub-ps pulses," Opt. Express 23, 13866-13879 (2015).

16. F. Zernike and H. C. Brinkman, "Hypersphärische funktionen und die in sphärische bereichen orthogonalen polynome," in Proc. Akad. Wet. Amsterdam, vol. 38 (1935), pp. 161-170.

17. M. Bellini and T. W. Hänsch, "Phase-locked white-light continuum pulses: toward a universal optical frequency-comb synthesizer," Opt. Lett. 25, 1049-1051 (2000).

18. A. Maréchal, "étude des effets combinés de la diffraction et des aberrations géométriques sur l'image d'un point lumineux," Revue d'optique, théorique et instrumentale 26, 257-277 (1947).

19. F. Zernike, "Beugungstheorie des Scheidenverfahrens und seiner verbesserten Form, der Phasenkontrastmethode," Phys. : Nederlandsch tijdschrift voor natuurkunde 1, 689-704 (1934). OCLC: 772363588.

20. A. Brodeur and S. L. Chin, "Ultrafast white-light continuum generation and self-focusing in transparent condensed media," J. Opt. Soc. Am. B 16, 637-650 (1999).

21. V. Kandidov, O. Kosareva, I. Golubtsov, W. Liu, A. Becker, N. Akozbek, C. Bowden, and S. Chin, "Self-transformation of a powerful femtosecond laser pulse into a white-light laser pulse in bulk optical media (or supercontinuum generation)," Appl. Phys. B: Lasers Opt. 77, 149-165 (2003).

22. M. Bradler, P. Baum, and E. Riedle, "Femtosecond continuum generation in bulk laser host materials with sub- $\mu \mathrm{j}$ pump pulses,” Appl. Phys. B 97, 561-574 (2009).

23. C. P. Hauri, J. Biegert, U. Keller, B. Schaefer, K. Mann, and G. Marowski, "Validity of wave-front reconstruction and propagation of ultrabroadband pulses measured with a Hartmann-Shack sensor," Opt. Lett. 30, 1563-1565 (2005). 